\title{
The Analysis of Flow Separation Loss of Impeller in Centrifugal Compressor under Variable Conditions
}

\author{
Bin Cai ${ }^{1, a}$, Yongsheng Su${ }^{2, b}$ \\ ${ }^{1}$ School of Mechanical and Power Engineering, East China University of Science and Technology, \\ Shanghai 200237, China; \\ ${ }^{2}$ School of Mechanical and Power Engineering, East China University of Science and Technology, \\ Shanghai 200237, China; \\ Acaibin24@163.com, byssu@ecust.edu.cn
}

Keywords: Angle of attack, Degree of separation loss, Equivalent volume, Numerical simulation

\begin{abstract}
In order to study the change condition of the influence on the centrifugal compressor blade field, we change the size of the centrifugal compressor inlet flow and the centrifugal compressor impeller rotational speed,using the realizable $\kappa-\varepsilon$ ii equation model to do the steady numerical simulation for the full port of centrifugal compressor. Through calculation, we get different inlet flow and rotate speed of centrifugal compressor internal flow field distribution of relative velocity, at the same time using the concept of Degree of Separation Loss to analyze the separation loss within impeller passage. The results show that under the condition of variable working condition, the change of Angle of attack is the primary factor for the the impeller separation, and when the Angle of attack of the same time, the smaller the relative velocity, the greater the separation loss. Therefore in the process of actual production, in order to reduce the loss of separation, we should reduce the size of Angle of attack, appropriatly increase import flow or impeller rotational speed.
\end{abstract}

\section{Introduction}

Centrifugal compressor occupies an important position in national economic sectors, and have a wide range of applications especially in the metallurgical, petrochemical, gas, refrigeration, and power industries ${ }^{[1]}$.

Most of the centrifugal compressor during actual operation are in a certain range of working conditions, very few working at a point. So, except improving the efficiency of the design point, improving the regulating performance of centrifugal compressor is also one of the effective ways to save energy. When the angle of attack of impeller inlet flow change, the separation and loss of impeller will change. So it is necessary to study under changing operation mode, the change of flow separation of internal loss when the impeller Angle of attack changes ${ }^{[2]}$.

For some years, on the entrance of the impeller flow angle of attack i, We have made many experiments on different types of impeller flow angle of attack effecting the performance of a single stage compressor ${ }^{[3]}$. But because of various concrete situation of test is not the same, the results are inconsistent. The biggest differences is the size of the positive and negative angle of attack effect on loss. One view show that in the case of positive angle of attack the blade inlet part will become invasive so that positive angle of attack will lead to the loss increase rapidly. The other view show that negative angle of attack will lead to the loss increase rapidly ${ }^{[4]}$.

\section{Calculation model}

The calculation model is one stage of a multistage centrifugal compressor. As shown in Figure 1, it is made up of import section, closed type centrifugal impeller, no leaves diffuser, bend and reflux device. The discharge coefficient, the Polytropic efficiency, the impeller blade outlet angle of the base stage. It belongs to bend after type arc impeller. The impeller blade number pieces and the reflux device blade number pieces. Both of them are the circular blade. The main geometry model of 
centrifugal compressor see table 1. Working speed, inlet temperature, inlet pressure, the working medium is air, the mass flow rate is. Compression ratio is. Figure 1 is a three-dimensional model of the compressor.

Table 1 Basic parameters of compression base model

\begin{tabular}{cc|cc}
\hline parameter & number & parameter & numeber \\
\hline Wheel Diameter/d & $0.125 \mathrm{~m}$ & Diffuser inlet diameter/ $D_{3}$ & $0.40 \mathrm{~m}$ \\
Impeller inlet diameter/ $D_{0}$ & $0.205 \mathrm{~m}$ & Diffuser outlet diameter/ $D_{4}$ & $0.635 \mathrm{~m}$ \\
Blade inlet diameter/ $D_{1}$ & $0.210 \mathrm{~m}$ & Width of diffuser inlet/ $\mathrm{b}_{3}$ & $14 \mathrm{~mm}$ \\
Width of blade inlet/ $b_{1}$ & $30.5 \mathrm{~mm}$ & Reflux device inlet diameter/ $D_{5}$ & $0.635 \mathrm{~m}$ \\
Width of impeller outlet/ $b_{2}$ & $16.36 \mathrm{~mm}$ & Reflux device outlet diameter/ $D_{6}$ & $0.208 \mathrm{~m}$ \\
Impeller outlet diameter/ $D_{2}$ & $0.38 \mathrm{~m}$ & Reflux device inlet installtion angle & 33 \\
Blade inlet installation angle & 29 & & \\
\hline
\end{tabular}

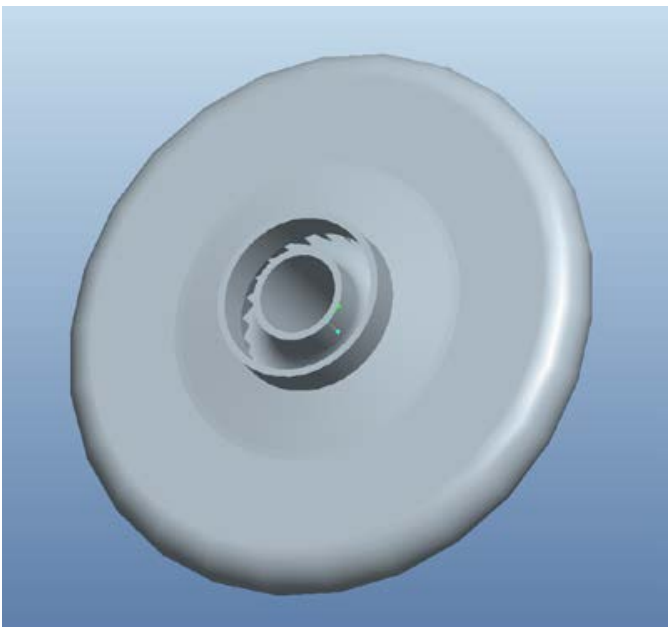

Figure1 the 3D model of the of the compressor stag

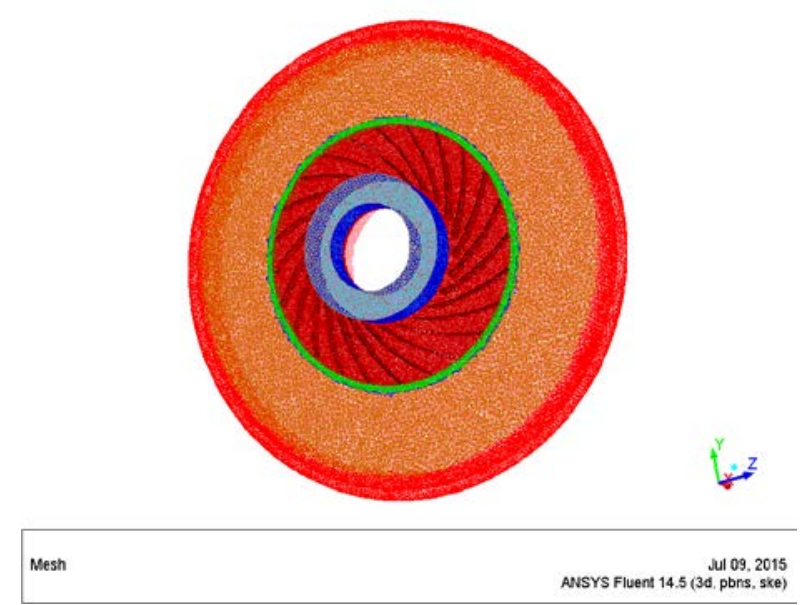

Figure 2 the wall mesh of the compressor stage

Because of the complicated geometrical structure of centrifugal compressor, this paper uses ICEM to mesh on it. The flow field is divided into different blocks and the non-uniform tetrahedral meshes are suitably set up for every block. Total grid number is 1133423 . The model is divided into three blocks, and the mesh density of the impeller is higher than the other two blocks. After checking, the minimum unit mesh quality is 0.3 , which can meet the requirements of the calculation. Figure 2 shows the 3D shape of the compressor stage and the wall mesh.

\section{Numerical calculation method}

In consideration that the paper is to analyze the flow field at different angle of attack, the flow field can be calculated by the steady compressible flow field. Reynold's averaged Navier-Stokes equation for steady compressible flow, together with standard $\kappa-\varepsilon$ double equation models, is solved by using SIMPIE algorithm for the time marching pressure coupled equations. The cell center finite volume method is used to solve N-S equations, in which the velocity, turbulent kinetic energy, turbulent energy dissipation rate, and energy are used in the two order upwind difference scheme with higher precision. In order to make the equation more easily convergence, the under relaxation factor of pressure, momentum, turbulent kinetic energy and turbulent dissipation rate are respectively cut to $0.2,0.5,0.5$ and 0.5 . The convergence scheme is based on the average residual error of all the control volume in the computational domain, which the convergence accuracy of the energy term is, and the convergence of the other terms is. 
The inlet boundary is given mass flow, and the outlet is given static pressure and no slip and adiabatic wall conditions are given in the enclosure wall. Multiple reference frame model is used for the dynamic and static coupling of the rotor impeller and stator inlet and diffuser. In this way, the complicated flow field of centrifugal compressor can be simplified as the instantaneous flow field in a rotating impeller, which fixed the unsteady problem.

\section{Result Analysis}

\section{1 analysis of relative velocity vector diagram}

Figure 3, 4, 5 respectively show when the inlet flow rate is $70 \%, 100 \%$ and $150 \%$, the distribution of the relative velocity of the centrifugal compressor in the $\mathrm{Z}=-10 \mathrm{~mm}$ section of the impeller blade surface. Comparison of figures $3,4,5$, as the flow rate is changed from small to large, the inlet flow velocity of the impeller increases gradually, and the angle of attack is from the positive to the negative. Figure 3 is the relative velocity distribution of the impeller at the time of $70 \%$ flow. From the picture, we can see that when the angle of attack is positive, there is a significant speed decrease in the non working surface of the impeller. Figure 4 is the relative velocity distribution of the impeller at the time of $100 \%$ flow. From the picture, we can see that when the angle of attack is 0 , the flow velocity distribution of impeller is uniform, and a small portion of the separation zone may occur in the outlet section of the impeller. Figure 4 is the relative velocity distribution of the impeller at the time of $150 \%$ flow. From the picture, we can see when the angle of attack is negative, there is a significant velocity decrease in the area of the working surface edge of the blade, and this area is gradually reduced along the outlet of the impeller. This is due to the inertia of the air flow, limiting the separation of the expansion.

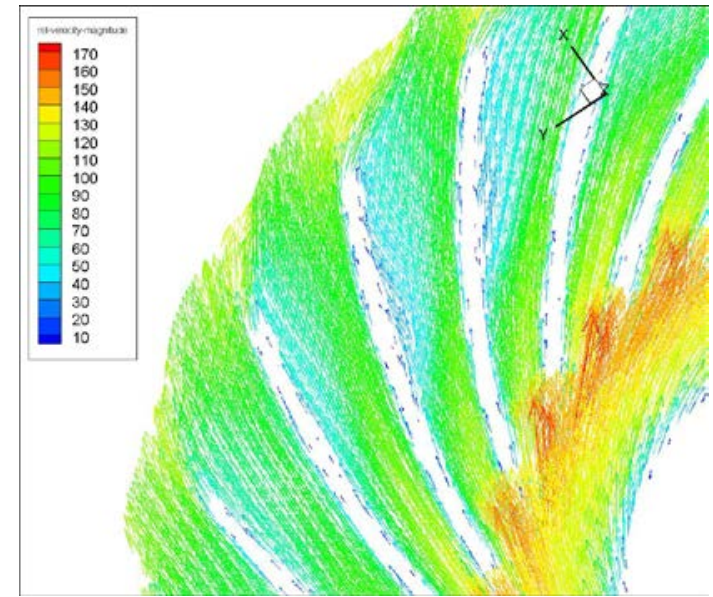

Figure 3 Relative velocity at $100 \%$ flow

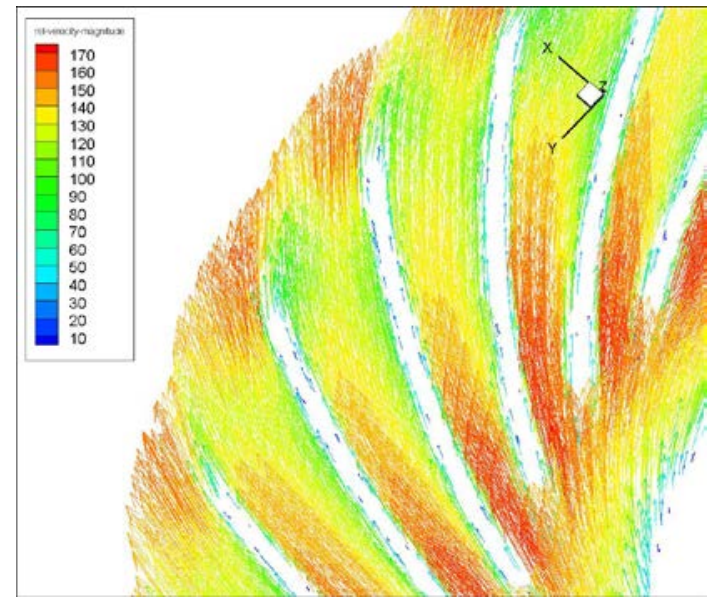

Figure 4 Relative velocity at $70 \%$ flow

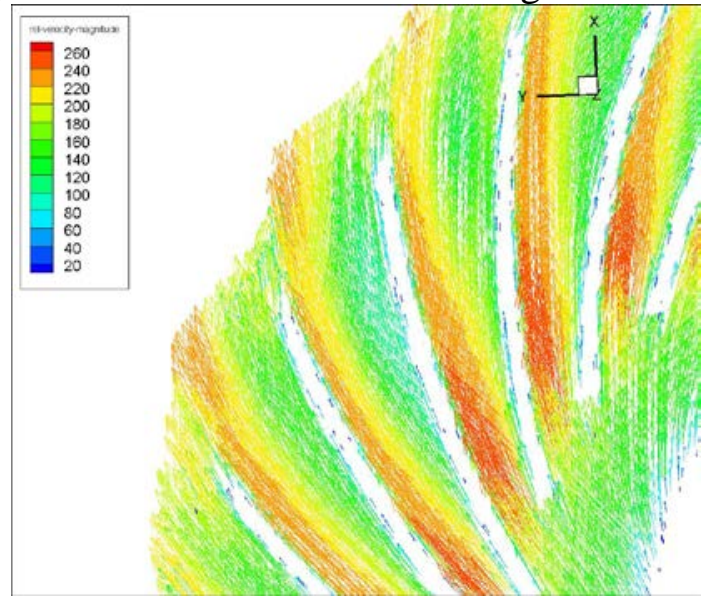

Figure 5 Relative velocity at $150 \%$ flow 


\subsection{Analysis on the separation loss of the impeller under different inlet flow}

Compressor running under variable working conditions which leads to the size and position of the velocity separation zone in the impeller are different is one of the reasons for the low efficiency of the centrifugal compressor. In order to do better statistics for the separation of the impeller flow channel with different operating conditions, We specifically lead to the degree of separation loss parameters $K_{\mathrm{sh}}$ :

$$
\begin{aligned}
& K_{\mathrm{sh}}=K_{1} \cdot V_{\mathrm{f}} / V_{\mathrm{z}} \\
& h_{\mathrm{sh}}=K_{\mathrm{sh}} \cdot h_{\mathrm{tot}}
\end{aligned}
$$

$V_{\mathrm{f}}$ in the equation (1) is the size of the equivalent volume of the separation zone in the flow passage of the impeller; $V_{\mathrm{z}}$ is the size of the total volume of the impeller; $K_{1}$ is proportional coefficient;

in the equation (2) $h_{s h}$ is the internal shock loss; $h_{\text {tot }}$ is the total energy delivered by the impeller to the gas.

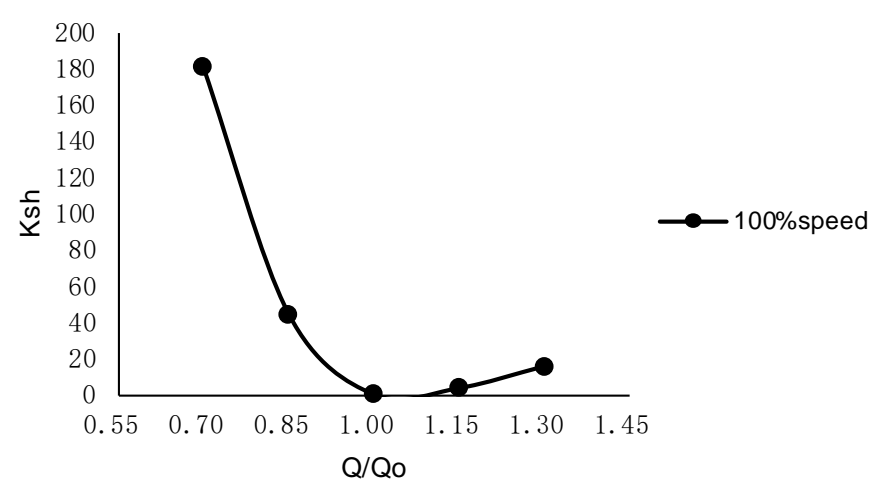

Figure 6 different $K_{\text {sh }}$ at different inlet flow rate

Figure 6 shows the different separation loss in the impeller at different inlet flow rate. From the diagram, the whole curve is concave. $K_{\mathrm{sh}}$ minimum near the design flow point, and the curve rise respectively from this point to the left and right. This shows that when the impeller angle of attack is near the 0-, the separation loss is minimum, and the separation loss due to the change of the impeller angle of attack is the minimum. It can also be observed that the separation loss degree at the flow less than the design flow is much greater than the separation loss degree at the flow more than the design flow at the same situation. This shows that when the flow is greater than the design flow, the expansion of the separation region is limited by the inertia force of the air flow to some extent.

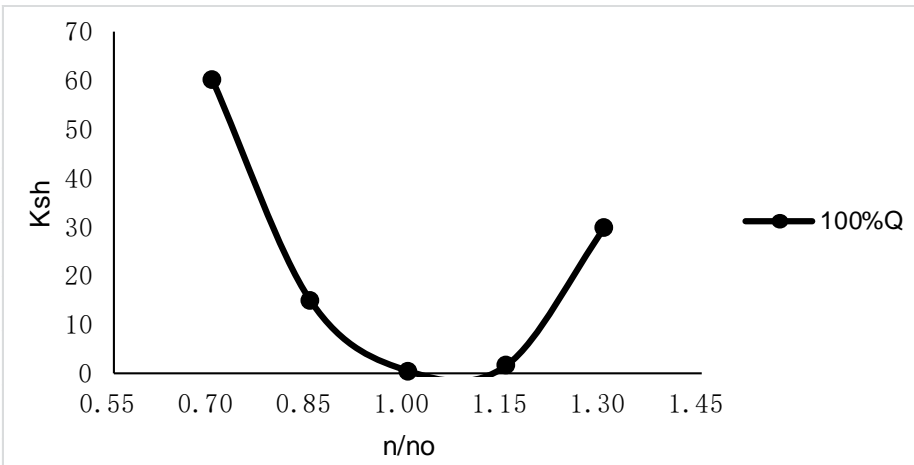

Figure 7 different $K_{\mathrm{sh}}$ at different rotational speed 
Figure 7 shows that in the case of the same inlet flow, the change of the separation loss degree of different impeller rotational speed. The change of impeller rotational speed changes the convected speed of the fluid, so as the rotational speed increases, the angle of attack is changed from positive to negative. This is just the opposite of the change in the flow(the angle of attack is changed from negative to positive as the rotational speed increases). From the figure it can be observed that with the increase of speed, the degree of separation loss is a process of increasing. At the same time can be obtained, when the angle of attack is less than 0 (the impeller rotational speed is larger), $K_{\mathrm{sh}}$ does not

appear to be a significant increase. Its growth trend is even slower than the condition when the angle of attack is positive. This is different from the situation in Figure 6. This shows that expect the angle of attack, the relative velocity of the air flow is an important factor to influence the separation loss.

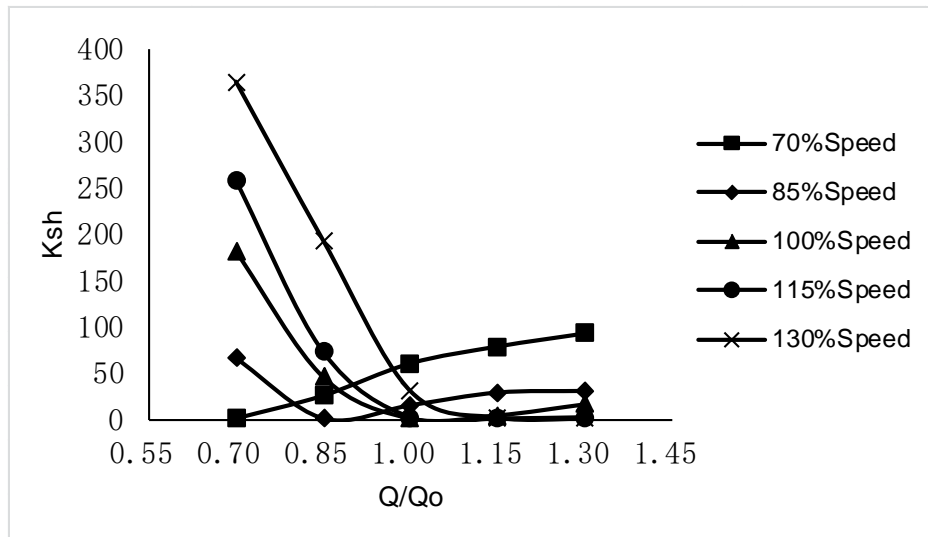

Figure 8 different $K_{\mathrm{sh}}$ at different inlet flow rate and rotational speed

Figure 8 shows the change in the degree of separation loss of the different inlet flow at each impeller rotational speed. As can be seen from the diagram, when the flow becomes large, the $K_{\mathrm{sh}}$

curve of different rotational speed is becoming more and more gentle. This shows that in the case of the same angle of attack. The smaller the inlet flow is, the greater the separation loss is. As can alse be seen from the diagram, when the flow rate and the impeller rotational speed of expansion (or reduction) the percentage of the same time, the separation of the loss is relatively small, and almost zero. This is because corresponding to each flow, there is a relative corresponding impeller rotational speed to lead the minimum $K_{\mathrm{sh}}$. At the same time, we notice that when the speed is large, and the

flow velocity is small, the separation of the loss degree is a significant growth process. This is because that working condition is close to the surge line of compressor impeller. So the speed of the sudden change, so as to degree of separation loss. It should be avoided in the actual operation of the compressor.

\section{Summary}

(1)The change of the angle of the impeller is the primary factor in the separation of the loss when the working condition is changed.

(2)In the case of equal angle of attack, the different relative velocity can also lead to different degrees of separation loss. The smaller the relative velocity, the greater the degree of separation loss.

(3)For the condition that the inlet flow is not up to the design flow rate, adjusting the speed to the corresponding working conditions can effectively reduce the separation loss and improve the efficiency of the whole flow passage. 


\section{References}

[1]Xu Z. Principle of centrifugal compressor[M]. Beijing: Machinery Industry Press. 2004.

[2]Cheng H, Wen SP, Xu ZX. Optimization design of small flow coefficient centrifugal compressor impeller[J]. Fan Technology. 2013(3): 43-46.

[3]Zhang SM, Guo PH, Wen XP et. CFD analysis and parameter optimization design of multi wing centrifugal fan[J]. Fan Technology, 2011(4): 40-43.

[4]Xing SK, Ma CC, Lv W. The influence of the change of the impeller angle of attack on the efficiency of the impeller[J]. Tech Review reports, 2013, 30(33): 27-30.

[5]Song CC, Su YS. Effect of Modification to Tongue and Basic Circle Diameter on Vibration in a Double-suction Centrifugal Pump[J]. Mechatronic[J]. 2015(2): 76-80.

[6]Wang Y, Liu HL, Yuan SQ, Tan MG, Wang K.Influence of blade inlet angle of attack on cavitation characteristics of centrifugal pump. Fluid Machinery. 2011, 39(4): 17-20, 66.

[7]Chen DM, Wu HY, Zhang CL. Analysis and Research on main aerodynamic design parameters of centrifugal compressor impeller. Fan Technology. 2006(3): 1-4.

[8]Liu ZG, Wang GL, Li JF. Effection of non-uniform entrance velocity profile on flow performance of centrifugal compressor stage. Journal of Engineering Thermophysics. 2009.3, 30(3): 415-418. 